\title{
Uso de álcool e tabaco entre universitários de Terapia Ocupacional de uma universidade pública*
}

\author{
Marina Coelho de Pinho ${ }^{1}$ \\ (D) https://orcid.org/0000-0002-6337-8321 \\ Rayane Cristina Faria de Souza ${ }^{1}$ \\ (D) https://orcid.org/0000-0001-9600-3270 \\ Flavia Batista Portugal ${ }^{2}$ \\ (D) https://orcid.org/0000-0002-4425-2627 \\ Marluce Mechelli de Siqueira² \\ (D) https://orcid.org/0000-0002-6706-5015
}

\footnotetext{
Artigo extraído da dissertação de mestrado "Uso de álcool e tabaco entre universitários de Terapia Ocupacional de uma universidade pública", apresentada à Universidade Federal do Espírito Santo, Vitória, ES, Brasil.

${ }^{1}$ Universidade Federal do Espírito Santo, Vitória, ES, Brasil.

2 Universidade Federal do Espírito Santo, Departamento de Enfermagem e Obstetrícia, Vitória, ES, Brasil.
}

\begin{abstract}
Objetivo: traçar o perfil do uso de álcool e tabaco e seus fatores associados entre universitários de Terapia Ocupacional. Método: estudo transversal realizado com estudantes acima de 18 anos do primeiro ao último período do curso de Terapia Ocupacional de uma universidade pública. O instrumento de pesquisa utilizado foi o questionário proposto pela Secretaria Nacional de Políticas sobre Drogas para o I Levantamento Nacional sobre o Uso de Álcool e Outras Drogas entre Universitários das 27 Capitais Brasileiras. Para a análise estatística, foi utilizado o Statistical Package for the Social Science. Resultados: a prevalência de "binge drinking no ano" foi de $61,5 \%$ e de "binge drinking no mês" foi de $46,8 \%$. O uso na vida de tabaco foi de $36 \%$ e no ano de $25,7 \%$. A religião mostrou-se significativamente associada ao "binge drinking no ano" e ao uso na vida e no ano de tabaco. "Pegar carona com o motorista da vez" e "Pegar carona com motorista alcoolizado" associaram-se significativamente às variáveis "binge drinking no ano" e "binge drinking no mês". Conclusão: os resultados contribuem para criar ações de prevenção ao uso indevido de álcool e tabaco, bem como à inclusão ou ampliação da abordagem do tema no currículo do curso.
\end{abstract}

Descritores: Estudantes; Tabaco; Bebidas Alcoólicas; Políticas Públicas; Prevenção \& Controle.

\section{Como citar este artigo}

Pinho MC, Souza RCF, Portugal FB, Siqueira MM. Use of alcohol and tobacco among university students of Occupational Therapy at a public university. SMAD, Rev Eletrônica Saúde Mental Álcool Drog. 2020;16(1):1-12. doi: https://dx.doi.org/10.11606/issn.1806-6976.smad.2020.152411 


\title{
Use of alcohol and tobacco among university students of Occupational Therapy at a public university
}

\begin{abstract}
Objective: outline the profile of alcohol and tobacco use and its associated factors among university students of Occupational Therapy. Method: cross-sectional study carried out with students over 18 years old, from the first to last period of Occupational Therapy course of a public university. The research instrument used was the questionnaire proposed by National Secretariat for Policy on Drugs for I National Survey on Use of Alcohol and Other Drugs among University Students of the 27 Brazilian Capitals. Statistical Package for the Social Science was used for statistical analysis. Results: the prevalence of "binge drinking in the year" was $61.5 \%$ and "binge drinking in the month" was $46.8 \%$. The use in life of tobacco was $36 \%$ and in year $25,7 \%$. Religion was significantly associated with "binge drinking in the year" and the use in life and in year of tobacco. "Taking a ride with the driver of the time" and "Taking a ride with an alcoholic driver" were significantly associated with "binge drinking in the year" and "binge drinking in the month". Conclusion: results contribute to create actions to prevent alcohol and tobacco abuse, as well the inclusion or extension of the approach to the topic in course curriculum.
\end{abstract}

Descriptors: Students; Tobacco; Alcoholic Beverages; Public Policies; Prevention \& Control.

\section{Uso de alcohol y tabaco entre universitarios de Terapia Ocupacional de una universidad pública}

Objetivo: trazar el perfil del uso de alcohol y tabaco y sus factores asociados entre universitarios de Terapia Ocupacional. Método: estudio transversal realizado con estudiantes mayores de 18 años, del primero al último período del curso de Terapia Ocupacional de una universidad pública. Instrumento de investigación utilizado fue cuestionario propuesto por Secretaría Nacional de Políticas sobre Drogas para el I levantamiento nacional sobre el uso de alcohol y otras drogas entre universitarios de las 27 capitales brasileñas. Para análisis estadístico se utilizó el Statistical Package for the Social Science. Resultados: prevalencia de "binge drinking en año" fue 61,5\% y de "binge drinking en mes" fue $46,8 \%$. El uso en la vida de tabaco fue del $36 \%$ y en año del 25,7\%. Religión se mostró significativamente asociada al "binge drinking en el año" y al uso en la vida y en año de tabaco. "Tomar carona con conductor de la vez" y "Tomar carona con conductor alcohólico", se asociaron significativamente a las variables "binge drinking en año" y "binge drinking en mes". Conclusión: resultados contribuyen a crear acciones de prevención al uso indebido de alcohol y tabaco, así como inclusión o ampliación del abordaje del tema en currículo del curso.

Descriptores: Estudiantes; Tabaco; Bebidas Alcohólicas; Políticas Públicas; Prevención y Control. 


\section{Introdução}

No decorrer da história da humanidade, é possível observar o uso das substâncias psicoativas (SPAs) em diferentes contextos (religiosos, de lazer ou terapêuticos). Trata-se de uma prática antiga, entretanto, atualmente, o consumo de SPAs tornou-se um claro problema devido à extensão do seu uso e às importantes consequências que causam para a saúde pública(1).

O uso indevido e descontrolado de SPAs tem sido cada vez maior e o aumento no consumo dessas substâncias ocorre, principalmente, entre os jovens(2). Com isso, há uma preocupação especial com uma grande parcela que compõe essa população: os universitários ${ }^{(3)}$. De acordo com o Instituto Nacional de Estudos e Estatísticas Educacionais Anísio Teixeira Inep, em 2016, essa população era composta por mais de oito milhões de universitários matriculados nos cursos de graduação ofertados pelas Instituições de Ensino Superior (IES) no Brasil(4).

Frente ao grande número de universitários no país, destaca-se, também, que esses utilizam mais bebidas alcoólicas, maconha, tranquilizantes, inalantes, alucinógenos e anfetamínicos quando comparados à população geral brasileira de mesma faixa etária(5).

Entre as diversas SPAs consumidas, não só por universitários, mas por toda a sociedade, o álcool e o tabaco merecem atenção, pois se tratam de substâncias lícitas, mas que podem causar danos à saúde tanto quanto outras substâncias( ${ }^{(6)}$.

Em sua fase inicial, o álcool pode causar sensações de euforia, desinibição, sociabilidade, prazer e alegria. Posteriormente, pode causar diminuição da autocrítica, lentificação psicomotora, redução dos reflexos, sonolência e prejuízos na capacidade de raciocínio e concentração. Em doses muito altas, o uso de álcool gera vômitos e insuficiência respiratória, podendo chegar à anestesia, coma e até mesmo morte ${ }^{(7)}$.

De uma forma geral, os universitários estão consumindo cada vez mais os diversos tipos de bebidas alcoólicas, tais como destilados, cervejas, bebidas ice e vinho $^{(8)}$. Nesse contexto, vale destacar um importante padrão de uso entre universitários: o binge drinking ou beber pesado episódico, o qual se caracteriza pelo consumo de cinco ou mais doses para homens e quatro ou mais doses para mulheres de bebidas alcoólicas em uma única ocasião de consumo. Conforme o I Levantamento Nacional sobre o Uso de Álcool e Outras Drogas entre Universitários das 27 Capitais Brasileiras, $25 \%$ dos universitários brasileiros referiram, pelo menos, uma ocorrência de binge drinking nos 30 dias anteriores à entrevista, e cerca de $33 \%$ relataram ter feito uso do álcool nesse padrão nos últimos 12 meses ${ }^{(9)}$.

Já o uso do tabaco de forma ativa e até mesmo passiva é considerado fator de risco para o desenvolvimento de diversas doenças, como câncer, doenças pulmonares e doenças cardiovasculares, sendo a maior causa de mortes evitáveis ${ }^{(10-11)}$. Segundo a Organização Mundial da Saúde (OMS), seu uso leva à morte de mais de sete milhões pessoas a cada ano. Mesmo sabendo de todas as doenças que podem ser desencadeadas em decorrência do uso de tabaco, em 2015, havia 1,1 bilhão de fumantes em todo o mundo(12). No Brasil, em 2016, o percentual total de fumantes com 18 anos ou mais era de $10,2 \%{ }^{(13)}$.

Dessa forma, as pesquisas são importantes ferramentas para entender melhor os padrões de consumo do álcool e tabaco, dando subsídios para o desenvolvimento de estratégias de prevenção e, consequentemente, reduzindo os problemas causados pelo seu uso indevido e os transtornos relacionados a ele ${ }^{(14)}$.

Diante disso, o objetivo deste trabalho foi traçar o perfil do uso de álcool e seus fatores associados entre os universitários do curso de Terapia Ocupacional de uma universidade pública.

\section{Método}

Trata-se de um estudo transversal, com caráter quantitativo. A população foi composta por estudantes de graduação do primeiro ao último período do curso de Terapia Ocupacional de uma universidade pública, com matrícula ativa no segundo semestre de 2016 e mais de 18 anos, totalizando 130 estudantes.

O instrumento utilizado foi o questionário proposto pela Secretaria Nacional de Políticas sobre Drogas (SENAD) para o I Levantamento Nacional sobre o Uso de Álcool e Outras Drogas entre Universitários das 27 Capitais Brasileiras, realizado em 2009. O instrumento de pesquisa é dividido em seções com a proposta de coletar dados socioeconômicos e demográficos, informações acadêmicas, atividades gerais, satisfação e desempenho acadêmico, consumo geral de drogas, consumo de tabaco e derivados, consumo de álcool, detalhamento do consumo de outras drogas e comportamentos gerais por meio de 72 questões ${ }^{(13)}$.

Na seção "consumo geral de drogas", a identificação do uso de SPAs foi feita conforme a classificação da Organização Mundial de Saúde: uso na vida, ou seja, "Pelo menos, uma vez na vida", nos últimos 12 meses ("Pelo menos, uma vez nos 12 meses anteriores à entrevista") e nos últimos 30 dias ("Pelo menos, uma vez nos 30 dias que antecederam a entrevista"). Na seção "consumo de álcool", foi possível identificar a ocorrência de binge drinking no ano e no mês.

Inicialmente, foi realizado um estudo piloto com os alunos do primeiro período do curso de Enfermagem da universidade com o objetivo de treinar os pesquisadores e verificar o tempo de duração de todo o procedimento de coleta. Apenas aqueles maiores de 18 anos, que estavam presentes em sala de aula no momento da aplicação e 
que aceitaram participar do estudo mediante a assinatura do Termo de Consentimento Livre e Esclarecido (TCLE), responderam ao instrumento. Posteriormente, deu-se início à coleta de dados no curso de Terapia Ocupacional, que foi realizada no período setembro de 2016 a julho de 2017, por meio de censo, em sala de aula, em horário agendado com os professores responsáveis. Para isso, os pesquisadores disponibilizaram dois ou mais envelopes, um para colocar os TCLEs assinados e os outros para armazenar os questionários.

Para a análise dos dados, utilizou-se o programa Statistical Package for the Social Science (SPSS), versão 22. Utilizou-se o teste qui-quadrado para verificar a associação entre as variáveis desfecho ("binge drinking no ano", "binge drinking no mês", "uso na vida de tabaco e derivados" e "uso no ano de tabaco e derivados") com as variáveis socioeconômicas e demográficas (sexo, faixa etária, estado civil, classe socioeconômica, religião, "morar com os pais", "morar com os amigos"), "Participação em projetos acadêmicos", "Frequência às aulas", "Frequentar o Centro Acadêmico (CA), Diretório Acadêmico (DA) e Grêmio". Apenas para as variáveis desfecho "binge drinking no ano" e "binge drinking no mês", verificou-se a associação com as variáveis independentes: "Dirigiu sob o efeito de álcool"; "Foi motorista da vez"; "Pegar carona com motorista alcoolizado" e "Pegar carona com motorista da vez", assumindo nível de significância $p \leq 0,05$. Foram calculadas, também, as razões de prevalência (RP) e seus intervalos de confiança (IC). Por fim, realizou-se uma análise multivariada por meio da regressão logística múltipla na qual foram incluídas somente as variáveis associadas ao desfecho com um nível de significância $\mathrm{p} \leq 0,1$.

Este estudo foi aprovado ( $n^{\circ} 1.566 .208$ ) pelo Comitê de Ética em Pesquisa (CEP) do Centro de Ciências da Saúde (CCS) da Universidade Federal do Espírito Santo (UFES), sendo conduzido de acordo com a Resolução no 466/2012 do Conselho Nacional de Saúde. Os questionários eram anônimos e de autopreenchimento. A aplicação foi realizada somente após a assinatura do TCLE por todos os alunos presentes em sala de aula no dia da aplicação do instrumento, acima de 18 anos e que aceitaram participar.

\section{Resultados}

Dos 130 estudantes matriculados no curso, $111(85,4 \%)$ responderam ao questionário. O perfil socioeconômico e demográfico da população em questão encontra-se na Tabela 1, observando-se que a maioria era do sexo feminino $(83,8 \%)$, possuía idade entre 18 e 24 anos $(83,8 \%)$, era solteira $(88,3 \%)$, pertencia à classe econômica B1/B2 $(50,4 \%)$ e era predominantemente composta por evangélicos/protestantes (36\%), seguidos pelos católicos $(35,1 \%)$.
Tabela 1 - Perfil socioeconômico e demográfico dos universitários de Terapia Ocupacional de uma universidade pública, Vitória, ES, Brasil, 2017

\begin{tabular}{|c|c|c|}
\hline Categoria & $\mathbf{N}$ & $\%$ \\
\hline \multicolumn{3}{|l|}{ Sexo } \\
\hline Feminino & 93 & 83,8 \\
\hline Masculino & 18 & 16,2 \\
\hline Total & 111 & 100 \\
\hline \multicolumn{3}{|l|}{ Faixa etária (em anos) } \\
\hline 18 a 24 & 93 & 83,8 \\
\hline 25 a 34 & 12 & 10,8 \\
\hline 35 ou mais & 6 & 5,4 \\
\hline Total & 111 & 100 \\
\hline \multicolumn{3}{|l|}{ Estado civil } \\
\hline Solteiro(a) & 98 & 88,3 \\
\hline Casado(a)/"Vive junto" & 9 & 8,1 \\
\hline Separado(a)/Divorciado(a) & 4 & 3,6 \\
\hline Total & 111 & 100 \\
\hline \multicolumn{3}{|l|}{ Classe Socioeconômica } \\
\hline $\mathrm{A} 1 / \mathrm{A} 2$ & 1 & 0,9 \\
\hline $\mathrm{B} 1 / \mathrm{B} 2$ & 56 & 50,4 \\
\hline $\mathrm{C} 1 / \mathrm{C} 2$ & 44 & 39,6 \\
\hline $\mathrm{D} / \mathrm{E}$ & 10 & 9 \\
\hline Total & 111 & 100 \\
\hline \multicolumn{3}{|l|}{ Religião } \\
\hline Evangélica/Protestante & 40 & 36,4 \\
\hline Católica & 39 & 35,5 \\
\hline Não tenho religião & 25 & 22,7 \\
\hline Outras & 6 & 5,4 \\
\hline Total & 110 & 100 \\
\hline
\end{tabular}

A Tabela 2 apresenta os padrões de uso de álcool e tabaco verificados na população estudada: $85,6 \%$ dos universitários relataram já ter feito uso na vida de álcool e 75,7\% relataram uso no ano. Já a prevalência do uso de álcool nos últimos 30 dias que antecederam a pesquisa foi de $62,3 \%$.

Tabela 2 - Padrões de uso de álcool e tabaco e derivados entre universitários de Terapia Ocupacional de uma universidade pública. Vitória, ES, Brasil, 2017

\begin{tabular}{lcc}
\hline \multicolumn{1}{c}{ Características } & N & $\%$ \\
\hline Uso na vida de álcool & & \\
Sim & 95 & 85,6 \\
Não & 16 & 14,4 \\
Total & 111 & 100 \\
Uso no ano de álcool & & \\
Sim & 81 & 75,7 \\
Não & 26 & 24,3 \\
Total & 107 & 100 \\
Uso no mês de álcool & & \\
Sim & 66 & 62,3 \\
Não & 40 & 37,7 \\
Total & 106 & 100 \\
\hline & & (continua...)
\end{tabular}


Tabela 2 - continuação

\begin{tabular}{|c|c|c|}
\hline Características & $\mathbf{N}$ & $\%$ \\
\hline \multicolumn{3}{|l|}{ Binge drinking no ano } \\
\hline Sim & 67 & 61,5 \\
\hline Não & 42 & 38,5 \\
\hline Total & 109 & 100 \\
\hline \multicolumn{3}{|l|}{ Binge drinking no mês } \\
\hline Não & 58 & 53,2 \\
\hline Sim & 51 & 46,8 \\
\hline Total & 109 & 100 \\
\hline \multicolumn{3}{|c|}{ Uso na vida de tabaco e derivados } \\
\hline Sim & 40 & 36 \\
\hline Não & 71 & 64 \\
\hline Total & 111 & 100 \\
\hline \multicolumn{3}{|c|}{ Uso no ano de tabaco e derivados } \\
\hline Sim & 26 & 25,7 \\
\hline Não & 75 & 74,3 \\
\hline Total & 101 & 100 \\
\hline \multicolumn{3}{|c|}{ Uso no mês de tabaco e derivados } \\
\hline Sim & 19 & 79,1 \\
\hline Não & 72 & 20,9 \\
\hline Total & 91 & 100 \\
\hline
\end{tabular}

A maioria $(61,5 \%)$ relatou beber em binge nos 12 meses que antecederam a pesquisa e $46,8 \%$ relataram beber em binge nos últimos 30 dias que antecederam a pesquisa.

Em relação ao uso de tabaco e derivados, 40 alunos (36\%) relataram já ter feito uso alguma vez na vida, $26(25,7 \%)$ relataram uso no ano e $19(20,9 \%)$, uso no mês.

$\mathrm{Na}$ Tabela 3, é possível observar o resultado da análise bivariada para as variáveis desfecho "binge drinking no ano" e "binge drinking no mês". Na Tabela 4, observa-se o resultado da análise bivariada para as variáveis desfecho "uso na vida de tabaco e derivados" e "uso no ano de tabaco e derivados". As variáveis associadas aos desfechos em um nível de significância menor ou igual a $10 \%$ e que foram inclusas na análise multivariada podem ser observadas na Tabela 5, apresentando apenas os resultados para as variáveis que se mantiveram significativamente associadas $(p \leq 0,05)$.

Quanto às variáveis socioeconômicas e demográficas, na análise bivariada, a religião apresentou associação com a variável desfecho "binge drinking no

Tabela 3 - Fatores associados à realização de binge drinking (no ano e no mês) entre universitários de Terapia Ocupacional de uma universidade pública por meio de análise bivariada. Vitória, ES, Brasil, 2017

\begin{tabular}{|c|c|c|c|c|c|c|}
\hline & \multicolumn{3}{|c|}{ Binge no ano } & \multicolumn{3}{|c|}{ Binge no mês } \\
\hline & $\mathbf{N}(\%)$ & $\mathrm{p}$ - valor & $\mathrm{RP}(\mathrm{IC})$ & $\mathbf{N}(\%)$ & p - valor & $\mathrm{RP}(\mathrm{IC})$ \\
\hline \multicolumn{7}{|c|}{ Características socioeconômicas e demográficas } \\
\hline \multicolumn{7}{|l|}{ Sexo } \\
\hline Feminino & $54(59,3)$ & 0,305 & $1,781(0,585-5,422)$ & $41(45,1)$ & 0,415 & $1,524(0,551-4,216)$ \\
\hline Masculino & $13(72,2)$ & & 1 & $10(55,6)$ & & 1 \\
\hline Total & $67(61,5)$ & & & $51(46,8)$ & & \\
\hline \multicolumn{7}{|l|}{ Faixa etária } \\
\hline 18 a 24 & $59(64,8)$ & 0,104 & $2,305(0,827-6,420)$ & $45(49,5)$ & 0,211 & $1,957(0,676-5,662)$ \\
\hline Acima de 25 & $8(44,4)$ & & 1 & $6(33,3)$ & & 1 \\
\hline Total & $67(61,5)$ & & & $51(46,8)$ & & \\
\hline \multicolumn{7}{|l|}{ Estado civil } \\
\hline $\begin{array}{l}\text { Solteiro(a) Separado(a), } \\
\text { Viúvo(a) }\end{array}$ & $65(64,4)$ & 0,053 & $5,417(1,039-28,241)$ & $50(49,5)$ & 0,065 & $6,863(0,814-57,824)$ \\
\hline Casado(a), vive junto(a) & $2(25)$ & & 1 & $1(12,5)$ & & 1 \\
\hline Total & $67(61,5)$ & & & $51(46,8)$ & & \\
\hline \multicolumn{7}{|l|}{ Classe socioeconômica } \\
\hline $\mathrm{A} / \mathrm{B} / \mathrm{C}$ & $62(62)$ & 0,731 & $1,305(0,330-5,164)$ & $47(47)$ & 1 & $1,108(0,281-4,372)$ \\
\hline $\mathrm{D} / \mathrm{E}$ & $5(55,6)$ & & 1 & $4(44,4)$ & & 1 \\
\hline Total & $67(61,5)$ & & & $51(46,8)$ & & \\
\hline \multicolumn{7}{|l|}{ Religião } \\
\hline Ter religião & $46(54,1)$ & 0,003 & $0,168(0,047-0,608)$ & $36(42,4)$ & 0,081 & $0,441(0,174-1,119)$ \\
\hline Não ter religião & $21(87,5)$ & & 1 & $15(62,5)$ & & 1 \\
\hline Total & $67(61,5)$ & & & $51(46,8)$ & & \\
\hline Sim & $49(60,5)$ & 0,722 & $0,851(0,349-2,076)$ & $37(45,7)$ & 0,693 & $0,841(0,356-1,988)$ \\
\hline Não & $18(64,3)$ & & 1 & $14(50,0)$ & & 1 \\
\hline Total & $67(61,5)$ & & & $51(46,8)$ & & \\
\hline
\end{tabular}


Tabela 3 - continuação

\begin{tabular}{|c|c|c|c|c|c|c|}
\hline & \multicolumn{3}{|c|}{ Binge no ano } & \multicolumn{3}{|c|}{ Binge no mês } \\
\hline & $\mathbf{N}(\%)$ & $p$-valor & $\mathrm{RP}(\mathrm{IC})$ & $\mathrm{N}(\%)$ & $p$ - valor & $\mathrm{RP}(\mathrm{IC})$ \\
\hline \multicolumn{7}{|l|}{ Morar com os amigos } \\
\hline Sim & $3(75,0)$ & 1 & $1,922(0,193-19,109)$ & $2(50,0)$ & 1 & $1,143(0,155-8,420)$ \\
\hline Não & $64(61,0)$ & & 1 & $49(46,7)$ & & 1 \\
\hline Total & $67(61,5)$ & & & $51(46,8)$ & & \\
\hline \multicolumn{7}{|c|}{ Informações referentes à universidade } \\
\hline \multicolumn{7}{|c|}{ Participar de projetos acadêmicos } \\
\hline Sim & $20(69,0)$ & 0,333 & $1,560(0,632-3,853)$ & $16(55,2)$ & 0,291 & $1,582(0,673-3,720)$ \\
\hline Não & $47(58,8)$ & & 1 & $35(43,8)$ & & 1 \\
\hline Total & $67(61,5)$ & & & $51(46,8)$ & & \\
\hline \multicolumn{7}{|l|}{ Frequência às aulas } \\
\hline Algumas vezes falto & $65(64,4)$ & 0,053 & $5,417(0,768-8,624)$ & $49(48,5)$ & 0,279 & $2,827(0,544-14,678)$ \\
\hline Nunca falto & $2(25)$ & & 1 & $2(25)$ & & 1 \\
\hline Total & $67(61,5)$ & & & $51(46,8)$ & & \\
\hline \multicolumn{7}{|c|}{ Frequentar CA, DA e Grêmio } \\
\hline Sim & $4(80,0)$ & 0,647 & $2,603(0,281-24,120)$ & $2(40,0)$ & 1 & $0,748(0,120-4,666)$ \\
\hline Não & $63(60,6)$ & & 1 & $49(47,1)$ & & \\
\hline Total & $67(61,5)$ & & & $51(46,8)$ & & \\
\hline \multicolumn{7}{|c|}{ Comportamentos de risco } \\
\hline \multicolumn{7}{|c|}{ Dirigir sob efeito de álcool } \\
\hline Sim & $2(100)$ & 0,522 & $1,646(1,414-1,917)$ & $2(100)$ & 0,217 & $2,184(1,777-2,684)$ \\
\hline Não & $65(60,7)$ & & 1 & $49(45,8)$ & & 1 \\
\hline Total & $67(61,5)$ & & & $51(46,8)$ & & \\
\hline \multicolumn{7}{|l|}{ Foi o motorista da vez } \\
\hline Sim & $10(83,3)$ & 0,124 & $3,509(0,729-16,884)$ & $8(66,7)$ & 0,143 & $2,512(0,709-8,902)$ \\
\hline Não & $57(58,8)$ & & 1 & $43(44,3)$ & & 1 \\
\hline Total & $67(61,5)$ & & & $51(46,8)$ & & \\
\hline \multicolumn{7}{|c|}{$\begin{array}{l}\text { Pegar carona com motorista } \\
\text { alcoolizado }\end{array}$} \\
\hline Sim & $21(91,3)$ & 0,001 & $9,130(2,015-41,373)$ & $20(87)$ & 0 & $11,828(3,253-43,005)$ \\
\hline Não & $46(53,5)$ & & 1 & $31(36)$ & & 1 \\
\hline Total & $67(61,5)$ & & & $51(46,8)$ & & \\
\hline \multicolumn{7}{|c|}{$\begin{array}{l}\text { Pegar carona com motorista da } \\
\text { vez }\end{array}$} \\
\hline Sim & $28(84,8)$ & 0,001 & $5,313(1,854-15,221)$ & $22(67,7)$ & 0,006 & $3,241(1,373-7,653)$ \\
\hline Não & $39(51,3)$ & & 1 & $29(38,2)$ & & 1 \\
\hline Total & $67(61,5)$ & & & $51(46,8)$ & & \\
\hline
\end{tabular}

ano", associação essa que se manteve após a realização da análise multivariada.

A variável "frequência às aulas" apresentou associação com a realização de "binge drinking no ano" após a inclusão na análise multivariada $(p \leq 0,1)$.

"Pegar carona com motorista alcoolizado" e "pegar carona com motorista da vez" associaram-se a beber em binge no ano e no mês na análise bivariada e mantiveram-se associadas na análise multivariada.

A religião também se apresentou associada ao "uso no ano de tabaco e derivados" na análise bivariada, associação essa que também se manteve após a análise multivariada. Após a inclusão no modelo de regressão $(p \leq 0,1)$, a religião apresentou associação significativa com o "uso na vida de tabaco e derivados".

"Morar com pais, padrastos ou outros familiares" apresentou associação significativa com o "uso na vida de tabaco e derivados" na análise bivariada. Porém, a associação não se manteve após a análise multivariada.

A "participação em projetos acadêmicos" apresentou associação com duas variáveis desfecho na análise bivariada "uso na vida de tabaco e derivados" e "uso no ano de tabaco e derivados", porém, após a realização da análise multivariada, a associação não se manteve. 
Tabela 4 - Fatores associados ao uso (na vida e no ano) de tabaco e derivados entre universitários de Terapia Ocupacional de uma universidade pública por meio de análise bivariada. Vitória, ES, Brasil, 2017

\begin{tabular}{|c|c|c|c|c|c|c|}
\hline \multirow{2}{*}{ Variável } & \multicolumn{3}{|c|}{ Uso na vida de Tabaco e derivados } & \multicolumn{3}{|c|}{ Uso no ano de Tabaco e derivados } \\
\hline & $\mathbf{N}(\%)$ & $p$ - valor & $\mathrm{RP}(\mathrm{IC})$ & $\mathbf{N}(\%)$ & $\mathrm{p}$ - valor & $\mathrm{RP}(\mathrm{IC})$ \\
\hline \multicolumn{7}{|c|}{ Características socioeconômicas e demográficas } \\
\hline \multicolumn{7}{|l|}{ Sexo } \\
\hline Feminino & $33(35,5)$ & 0,783 & $1,157(0,410-3,268)$ & $23(27,1)$ & 0,756 & $0,622(0,162-2,384)$ \\
\hline Masculino & $7(38,9)$ & & 1 & $3(18,8)$ & & 1 \\
\hline Total & $40(36)$ & & & $26(25,7)$ & & \\
\hline \multicolumn{7}{|l|}{ Faixa etária } \\
\hline 18 a 24 & $31(33,3)$ & 0,178 & $0,5(0,18-1,386)$ & $22(25,9)$ & 1 & $1,048(0,306-3,589)$ \\
\hline 25 ou mais & $9(50)$ & & 1 & $4(25)$ & & 1 \\
\hline Total & $40(36)$ & & & $26(25,7)$ & & \\
\hline \multicolumn{7}{|l|}{ Estado civil } \\
\hline Solteiro(a), Separado(a), Viúvo(a) & $36(35,3)$ & 0,72 & $0,682(0,172-2,7)$ & $26(28,3)$ & 0,107 & $0,717(0,631-0,816)$ \\
\hline Casado(a), vive junto & $4(44,4)$ & & 1 & $0(0)$ & & 1 \\
\hline Total & $40(36)$ & & & $26(25,7)$ & & \\
\hline \multicolumn{7}{|l|}{ Classe econômica } \\
\hline $\mathrm{A} / \mathrm{B} / \mathrm{C}$ & $26(35,6)$ & 0,745 & $0,831(0,220-3,138)$ & $23(24,7)$ & 0,421 & $0,548(0,121-2,471)$ \\
\hline $\mathrm{D} / \mathrm{E}$ & $4(40)$ & & 1 & $3(37,5)$ & & 1 \\
\hline Total & $40(36)$ & & & $26(25,7)$ & & \\
\hline \multicolumn{7}{|l|}{ Religião } \\
\hline Ter religião & $27(31,4)$ & 0,059 & $0,422(0,171-1,047)$ & $15(19,5)$ & 0,01 & $0,286(0,107-0,763)$ \\
\hline Não ter religião & $13(52)$ & & 1 & $11(45,8)$ & & 1 \\
\hline Total & $40(36)$ & & & $26(25,7)$ & & \\
\hline \multicolumn{7}{|l|}{$\begin{array}{l}\text { Morar com pais, padrastos, outros } \\
\text { familiares }\end{array}$} \\
\hline Sim & $25(30,5)$ & 0,041 & $0,409(0,172-0,974)$ & $16(21,6)$ & 0,117 & $0,469(0,180-1,222)$ \\
\hline Não & $15(51,7)$ & & 1 & $10(37)$ & & 1 \\
\hline Total & $40(36)$ & & & $26(25,7)$ & & \\
\hline \multicolumn{7}{|l|}{ Morar com amigos } \\
\hline Sim & $3(75,0)$ & 0,132 & $5,676(0,570-56,495)$ & $3(75)$ & 0,051 & $9,652(0,957-97,341)$ \\
\hline Não & $37(34,6)$ & & 1 & $23(23,7)$ & & 1 \\
\hline Total & $40(36)$ & & & $26(25,7)$ & & \\
\hline \multicolumn{7}{|c|}{ Informações referentes à universidade } \\
\hline \multicolumn{7}{|l|}{ Participação em projetos acadêmicos } \\
\hline Sim & $15(51,7)$ & 0,041 & $2,443(1,027-5,813)$ & $12(41,4)$ & 0,023 & $2,924(1,140-7,499)$ \\
\hline Não & $25(30,5)$ & & 1 & $14(19,4)$ & & 1 \\
\hline Total & $40(36)$ & & & $26(25,7)$ & & \\
\hline \multicolumn{7}{|l|}{ Frequência às aulas } \\
\hline Algumas vezes falta & $3(37,5)$ & 1 & $1,070(0,242-4,734)$ & $2(25)$ & 1 & $0,958(0,181-5,072)$ \\
\hline Nunca falta & $37(35,9)$ & & 1 & $24(25,8)$ & & 1 \\
\hline Total & $40(36)$ & & & $26(25,7)$ & & \\
\hline \multicolumn{7}{|l|}{ Frequentar CA, DA e Grêmio } \\
\hline Sim & $19(47,5)$ & 0,059 & $2,154(0,965-4,809)$ & $12(31,6)$ & 0,297 & $1,615(0,653-3,997)$ \\
\hline Não & $21(29,6)$ & & 1 & $14(22,2)$ & & 1 \\
\hline Total & $40(36)$ & & & $26(25,7)$ & & \\
\hline
\end{tabular}

Tabela 5 - Fatores associados à realização de binge drinking (no ano e no mês) e ao uso (na vida e no ano) de tabaco e derivados entre universitários de Terapia Ocupacional de uma universidade pública por meio da análise multivariada. Vitória, ES, Brasil, 2017

\begin{tabular}{|c|c|c|c|c|c|c|}
\hline \multirow{2}{*}{ Variáveis* } & \multicolumn{3}{|c|}{ Binge no ano } & \multicolumn{3}{|c|}{ Binge no mês } \\
\hline & N (\%) & $p$ - valor & $\mathrm{RP}(\mathrm{IC})$ & $\mathbf{N}(\%)$ & p - valor & $\mathrm{RP}(\mathrm{IC})$ \\
\hline \multicolumn{7}{|l|}{ Religião } \\
\hline Ter religião & $46(54,1)$ & 0,002 & $0,062(0,011-0,354)$ & & & \\
\hline Não ter religião & $21(87,5)$ & & 1 & & $\dagger$ & \\
\hline Total & $67(61,5)$ & & & & & \\
\hline \multicolumn{7}{|c|}{$\begin{array}{l}\text { Pegar carona com motorista } \\
\text { alcoolizado }\end{array}$} \\
\hline Sim & $21(91,3)$ & 0,009 & $9,736(1,785-53,108)$ & $20(87)$ & 0,001 & $10,215(2,712-38,477)$ \\
\hline Não & $46(53,5)$ & & 1 & $31(36)$ & & 1 \\
\hline Total & $67(61,5)$ & & & $51(46,8)$ & & \\
\hline
\end{tabular}


Tabela 5 - continuação

\begin{tabular}{|c|c|c|c|c|c|c|}
\hline \multirow{2}{*}{ Variáveis* } & \multicolumn{3}{|c|}{ Binge no ano } & \multicolumn{3}{|c|}{ Binge no mês } \\
\hline & $\mathrm{N}(\%)$ & $p$ - valor & $\mathrm{RP}(\mathrm{IC})$ & $\mathrm{N}(\%)$ & $p$ - valor & $\mathrm{RP}(\mathrm{IC})$ \\
\hline \multicolumn{7}{|c|}{$\begin{array}{l}\text { Pegar carona com motorista } \\
\text { da vez }\end{array}$} \\
\hline Sim & $28(84,8)$ & 0,001 & $6,642(2,085-21,164)$ & $22(67,7)$ & 0,018 & $3,203(1,216-8,433)$ \\
\hline Não & $39(51,3)$ & & 1 & $29(38,2)$ & & 1 \\
\hline Total & $67(61,5)$ & & & $51(46,8)$ & & \\
\hline \multicolumn{7}{|l|}{ Frequência às aulas } \\
\hline Algumas vezes falta & $65(64,4)$ & 0,01 & $31,145(2,265-428,177)$ & & & \\
\hline Nunca falta & $2(25)$ & & 1 & & $\dagger$ & \\
\hline \multirow[t]{2}{*}{ Total } & $67(61,5)$ & & & & & \\
\hline & \multicolumn{3}{|c|}{ Uso na vida de tabaco e derivados } & \multicolumn{3}{|c|}{ Uso no ano de tabaco e derivados } \\
\hline \multicolumn{7}{|l|}{ Religião } \\
\hline Sim & $27(31,4)$ & 0,049 & $0,389(0,152-0,997)$ & $15(19,5)$ & 0,004 & $0,185(0,059-0,574)$ \\
\hline Não & $13(52)$ & & 1 & $11(45,8)$ & & 1 \\
\hline Total & $40(36)$ & & & $26(25,7)$ & & \\
\hline
\end{tabular}

*Somente variáveis que apresentaram associação menor ou igual a 5\% no modelo de regressão logística; ${ }^{\dagger}$ Variáveis não incluídas no modelo de regressão logística ou que não mantiveram associação significativa com a variável desfecho correspondente no modelo de regressão logística.

\section{Discussão}

A predominância do sexo feminino, da faixa etária de 18 a 24 anos, da classe econômica B1/B2 e de estudantes solteiros corrobora a maioria dos estudos realizados em cursos da área da saúde(15-22).

Apesar de a porcentagem encontrada para evangélicos/protestantes e católicos ter sido bem próxima, a predominância da religião evangélica/ protestante diverge da maioria dos estudos realizados com universitários em que a religião predominante é a religião católica, como mostra o resultado de uma pesquisa realizada em um campus universitário da área de Ciências Biológicas e da Saúde em Minas Gerais(23).

Em relação aos padrões de uso de álcool pela população estudada, os dados encontrados neste estudo corroboram os encontrados em pesquisas realizadas na UFES(19-22,24-25). Dados também encontrados no I Levantamento Nacional sobre o Uso de Álcool e Outras Drogas entre Universitários das 27 Capitais Brasileiras, em que se observou que $86,2 \%$ dos universitários já fizeram uso de álcool alguma vez na vida e $72 \%$, no ano(13). A prevalência do uso de álcool nos últimos 30 dias que antecederam a pesquisa corrobora estudo também realizado na UFES entre universitários de Farmácia(22).

Os resultados encontrados para beber em binge nos 12 meses e nos últimos 30 dias que antecederam a pesquisa mostraram-se superiores aos do estudo de referência nacional (I Levantamento Nacional sobre o Uso de Álcool, Tabaco e Outras Drogas entre Universitários das 27 Capitais Brasileiras), que mostrou que cerca de 33,3\% dos universitários brasileiros referiram, pelo menos, uma ocasião de beber em binge nos últimos 12 meses e $25 \%$ relataram ter feito uso do álcool nesse padrão nos 30 dias anteriores à entrevista(13), mostrando, assim, o quão preocupantes são os dados encontrados neste estudo e a importância de se trabalhar com a temática entre universitários.

Em relação aos padrões de uso de tabaco e derivados, em estudo realizado com os acadêmicos do curso de Odontologia de uma universidade privada de Curitiba, o uso na vida relatado foi um pouco superior ao encontrado neste estudo: $51,74 \%$ dos alunos relataram ter experimentado algum produto do tabaco, ao menos, uma vez ${ }^{(26)}$. Em estudo realizado entre estudantes do curso de Enfermagem da Universidade de Passo Fundo, o valor encontrado para o uso na vida também se mostrou um pouco superior, no qual 48,9\% dos estudantes relataram já ter feito uso na vida de tabaco. Nesse mesmo estudo, em relação ao uso no ano $(29,7 \%)$ e no mês $(20 \%)$, os resultados encontrados foram semelhantes aos encontrados neste estudo(27). Corroboram-se, ainda, por tais achados, os resultados mostrados no I Levantamento Nacional sobre o Uso de Álcool, Tabaco e Outras Drogas entre Universitários das 27 Capitais Brasileiras, no qual se observou: na vida de $46,7 \%$, uso no ano de $27,8 \%$ e uso no mês de $21,6 \%{ }^{(13)}$.

A associação encontrada entre religião e a variável desfecho binge drinking no ano sugere que, entre os universitários pesquisados que não possuem uma religião, há uma maior chance de encontrar alunos que beberam pesado de forma episódica nos últimos 12 meses.

A associação entre o uso de álcool e a religião pode ser encontrada em alguns estudos. Entre os usuários de uma clientela atendida na Clínica Saúde da Família, no Rio de Janeiro, os indivíduos que relataram baixa frequência de visitas a igrejas/templos (anual/nunca vão) tiveram, aproximadamente, três vezes mais chances de apresentar maior consumo de álcool quando comparados àqueles que frequentavam igrejas/templos com maior assiduidade (semanal ou mensal)(28). 
Da mesma forma, em estudo realizado com acadêmicos de uma Instituição de Ensino Superior (IES) na cidade de Montes Claros, observou-se que a chance de praticar o binge drinking foi 4,3 vezes maior entre os estudantes que não possuíam religião ou não se manifestaram como católicos ${ }^{(29)}$.

E, ainda, em estudo realizado com estudantes da área da saúde de uma universidade pública no norte de Minas, a falta de vínculo religioso foi uma variável estatisticamente associada à prática do consumo de risco de bebidas alcoólicas(24).

A relação entre o consumo de álcool por universitários e o comportamento religioso é um tema, ainda, pouco pesquisado, complexo e de difícil mensuração, porém, trata-se de um tema importante. Para entender melhor como os aspectos religiosos influenciam o uso de SPAs por universitários, é preciso ir além de conhecer aspectos como índices do uso, afiliação religiosa e frequência às reuniões/cultos de cunho religioso(30).

A associação encontrada entre a variável "frequência às aulas" e a realização de "binge drinking no ano" após a inclusão na análise multivariada sugere que, entre os universitários pesquisados que faltam às aulas, há uma maior chance de encontrar alunos que realizaram binge drinking nos últimos 12 meses que antecederam a pesquisa.

Em estudo realizado entre estudantes universitários também se observou essa associação, mostrando que, quando há abuso ou dependência de substâncias psicoativas, se destacam as faltas e os atrasos nas aulas e baixo desempenho acadêmico(31). Em uma instituição de ensino superior de Montes Claros, uma pesquisa realizada com acadêmicos regularmente matriculados nos cursos de graduação em Ciências Biológicas, Biomedicina, Enfermagem, Farmácia e Psicologia mostrou que a prática do binge drinking foi maior entre os que frequentemente não compareciam às atividades acadêmicas ${ }^{(29)}$.

Faltar às aulas/evasão escolar é um importante marcador de risco para o uso de SPAs. Em estudo realizado entre adolescentes, observou-se, ainda, um gradiente efeito dose-resposta: quanto mais os estudantes faltam às aulas, maior o risco de uso de SPAs(32).

"Pegar carona com motorista alcoolizado" e "pegar carona com motorista da vez" associaram-se a beber em binge no ano e no mês na análise bivariada e mantiveram-se na análise multivariada, sugerindo que, entre os alunos pesquisados que já tenham pegado carona com um motorista alcoolizado ou com o motorista da vez, há uma maior chance de encontrar universitários que beberam pesado e de forma episódica no último mês e no último ano.

Apesar de a informação sobre a associação de consumo de bebidas alcoólicas e direção poder resultar em acidente, um estudo realizado no Brasil mostrou que o uso de carona com condutores que tinham consumido alguma quantidade de bebida alcoólica era usual para $45,2 \%$ dos entrevistados. Entre jovens de 18 a 24 anos, o estudo mostrou que $66,1 \%$ relataram utilizar carona nessa condição(33).

Ao corroborar tal achado, em estudo transversal realizado com estudantes do sexo feminino de duas universidades do Nordeste brasileiro, a associação entre a realização de binge drinking e pegar carona com motorista alcoolizado também foi significativa(34).

Outra pesquisa realizada com universitários mostrou que aqueles que consumiram em binge drinking nos últimos três meses tiveram maior chance de relatar consequências ou problemas associados ao uso de álcool, entre eles, pegar carona com alguém que consumiu bebidas alcoólicas ${ }^{(35)}$.

A associação encontrada entre religião e as variáveis desfecho uso (na vida e no ano) de tabaco e derivados sugere que, entre os universitários pesquisados que não possuem religião, há uma maior chance de encontrar estudantes que já fizeram uso na vida e no ano de tabaco e derivados. Apesar de também ser um tema complexo e de difícil mensuração, na literatura, a associação da religião com o uso de tabaco e derivados pode ser observada em diferentes estudos realizados em diversas populações, como mostra uma pesquisa realizada entre fumicultores do município de São Lourenço do Sul, onde se observou que participar de atividades religiosas foi fator de proteção para o tabagismo (RP:0,69)(36).

Entrevistas realizadas com 383 pessoas, com mais de 18 anos, na favela de Paraisópolis, localizada em São Paulo, mostraram que a frequência religiosa mais alta foi associada a uma menor prevalência de tabagismo(37). Em estudo de corte prospectivo multicêntrico, projetado para descrever a evolução dos fatores de risco de doença cardíaca coronariana em adultos jovens e identificar hábitos, comportamentos e estilos de vida associados, observou-se que, entre os fumantes, aqueles que frequentaram menos serviços religiosos relataram fumar um número maior de cigarros por dia do que os frequentadores mais assíduos ${ }^{(38)}$. Entre estudantes do Ensino Médio da rede pública no Estado de Pernambuco, o adolescente que se considerava praticante de uma religião teve menor chance de relatar exposição ao consumo de bebidas alcoólicas (razão de chances, OR=0,71; IC95\%: 0,60 a 0,83) e ao tabagismo $(\mathrm{OR}=0,61 ; \text { IC95\%: } 0,46 \text { a } 0,79)^{(39)}$.

Ao se aderir a uma denominação religiosa e padrões de religiosidade, encontra-se um conjunto de valores, símbolos, comportamentos e práticas sociais que inclui, entre outras coisas, a aceitação ou a recusa ao uso de tabaco ou outras SPAs( ${ }^{(40)}$.

Morar com pais, padrastos ou outros familiares apresentou associação significativa com o uso na vida 
de tabaco e derivados na análise bivariada, sugerindo que, entre os universitários pesquisados que moram com os pais, padrastos ou outros familiares, há uma menor chance de encontrar estudantes que já fizeram uso de tabaco e derivados alguma vez na vida. Apesar da não manutenção da associação após a análise multivariada, estudos apontam tal associação. Em estudo realizado em uma universidade particular com alunos dos cursos da Escola de Saúde, observou-se que, entre os participantes que nunca utilizaram produtos derivados do tabaco, 57\% relataram que a família teve influência na não utilização e $43 \%$ indicaram que a família não teve influência na não utilização de produtos derivados do tabaco(41). De acordo com a Pesquisa Nacional de Saúde do Escolar (PeNSE), morar com os pais (pai e/ou mãe) foi fator protetor para o tabagismo(32). A literatura aponta, também, a importância da relação entre irmãos, principalmente, para meninas, na relação com irmãs mais velhas. O exemplo das irmãs é tão importante quanto o dos pais, pois elas têm grande responsabilidade de passar modelos saudáveis para essas meninas ${ }^{(42)}$.

O papel da família é essencial na promoção da saúde, que não é a única influência para o desenvolvimento e/ ou proteção ao uso de substâncias, mas exerce um papel importante ${ }^{(43)}$.

Na análise bivariada, a "participação em projetos acadêmicos" apresentou associação com as duas variáveis desfecho: "uso na vida de tabaco e derivados" e "uso no ano de tabaco e derivados". Porém, após a realização da análise multivariada, a associação não se manteve. Apesar da não manutenção da associação, estudos apontam que a sobrecarga de atividades acadêmicas pode levar a um excesso de preocupação, fato que contribui para o aparecimento de, entre outras coisas, distúrbios de sono, ansiedade e estresse ${ }^{(44)}$, e o estresse é visto como um fator associado ao tabagismo(45).

\section{Conclusão}

Os resultados encontrados dão subsídios para a criação de ações, políticas e programas de prevenção do uso indevido de álcool e tabaco entre universitários. Uma forma de prevenção, além de políticas e programas, seria a inclusão e/ou ampliação do tema na matriz curricular. Ao conhecer quais são os fatores que estão mais fortemente associados ao uso dessas SPAs, é possível aprofundar o estudo e, de forma mais assertiva, elaborar e transmitir informação aos universitários, visando sempre à prevenção e ao aumento de conhecimento dessa população em relação ao uso indevido de SPAs.

Por se tratar de uma abordagem transversal, não é possível estabelecer correlações entre o consumo de álcool e do tabaco com as variáveis associadas a eles, bem como não se pode generalizar os resultados para toda a população. Além disso, são necessários mais estudos para compreender, com nitidez, os resultados e as associações encontrados neste trabalho.

\section{Agradecimentos}

Ao Centro de Estudos e Pesquisa sobre Álcool e outras Drogas (CEPAD) pela oportunidade de realizar a pesquisa e aos integrantes do projeto Perfil do Uso de Substâncias Psicoativas entre universitários (PUSPA) pelo auxilio na realização desta pesquisa.

\section{Referências}

1. Gabatz RIB, Johann M, Terra MG, Mello SMP, da Silva AA, Brum JL. Percepção do usuário sobre a droga em sua vida. Escola Anna Nery. 2013;17(3):520-525. doi: 10.1590/S1414-81452013000300016.

2. Valença $C N$, Brandão ICA, Germano RM, de Vilar RLA, Monteiro AI. Abordagem da dependência de substancias psicoativas na adolescência: reflexão ética para a enfermagem. Escola Anna Nery. Revista de Enfermagem. 2013;17(3):562-567. doi: 10.1590/ S1414-81452013000300022.

3. Oliveira LG, Barroso LP, Wagner GA, Ponce JC, Malbergier A, Stempliuk VA et al. Drug consumption among medical students in São Paulo, Brazil: influences of gender and academic year. Revista Brasileira de Psiquiatria. 2009;31(3):227-239. doi: 10.1590/ S1516-44462009000300008

4. Instituto Nacional de Estudos e Pesquisas. Censo de educação superior 2016 - Principais resultados. Disponível em: http://download.inep.gov.br/educacao_ superior/censo_superior/documentos/2016/censo_ superior_tabelas.pdf. Acesso em 15 jan. 2018

5. Eckschmidt F, Andrade AG, Oliveira LG. Comparison of drug use between Brazilian and American college students and young Brazilian general population. Jornal Brasileiro de Psiquiatria. 2013;62(3):199-207. doi: 10.1590/S0047-20852013000300004

6. Paduani GF, Barbosa GA, Morais JCR de, Pereira JCP, Almeida MF, Prado MM, et al. Consumo de álcool e fumo entre os estudantes da Faculdade de Medicina da Universidade Federal de Uberlândia. Rev Bras Educ Médica. 2008;32(1):66-75. doi: 10.1590/ S0100-55022008000100009

7. Duarte PCAV, Formigoni MLOS. Álcool: efeitos agudos e crônicos. In: Secretaria Nacional de Políticas sobre Drogas. Efeitos de substâncias psicoativas: módulo 2. Brasília: Supera; 2017

8. Silva TC, Santos LBR, Salgado MHV. Alcoolismo: um estudo entre os universitários das instituições privadas de ensino de Guarulhos. Reva Eniac Pesquisa. 2017;6(1):81-92. doi: 10.22567/rep.v6i1.428 
9. Andrade A G, Duarte P C A V, Oliveira L G (Org.) I Levantamento Nacional sobre o Uso de Álcool e Outras Drogas entre Universitários das 27 Capitais Brasileiras. Observatório Brasileiro de Informações sobre Drogas, Faculdade de Medicina da Universidade de São Paulo, Secretaria Nacional de Políticas sobre Drogas. Brasília; 2010. 284 p.

10. Goulart D, Engroff P, Ely L. S. Sgnaolin V, Santos E F, Terra N L et al. Tabagismo em idosos. Rev Bras Geriatria Gerontol. 2010; 13(2):313-20. doi: 10.1590/ S1809-98232010000200015.

11. World Health Organization. WHO report on the global tobacco epidemic, 2011: warning about the dangers of tobacco. Geneva; 2011.

12. World Health Organization. Monitoring tobacco use and prevention policies. Geneva. 2017.

13. Brasil. Vigitel Brasil 2016: vigilância de fatores de risco e proteção para doenças crônicas por inquérito telefônico. 2017, 162p

14. Andrade AG, Anthony JC, Silveira CM. Álcool e suas consequências: uma abordagem multiconceitual. Barueri, SP: Minha Editora. 2009.

15. Chiapetti N, Serbena CA. Uso de álcool, tabaco e drogas por estudantes da área de saúde de uma Universidade de Curitiba. Psicologia: Reflexão e Crítica. 2007;20(2). doi: 10.1590/S010279722007000200017.

16. Pelicioli M. Perfil do consumo de álcool e prática do beber pesado episódico entre universitários brasileiros da área da saúde. Jornal Brasileiro de Psiquiatria. 2017; 66(3):150-6. doi: 10.1590/0047-2085000000164.

17. Pedrosa AAS, Camacho LAB, Passos SRL, Oliveira RVC. Consumo de álcool entre estudantes universitários. Caderno de Saúde pública. 2011; 27(8):1611-21. doi: $10.1590 /$ S0102-311X2011000800016

18. Ramis TR, Mielke GI, Habeyche EC, Oliz MM, Azevedo MR, Hallal PC. Tabagismo e consumo de álcool em estudantes universitários: prevalência e fatores associados. Revista Brasileira de Epidemiologia. 2012; 15(2):376-85. doi: 10.1590/S1415-790X2012000200015 19. Mardegan PS, Souza RS, Buaiz V, Siqueira MM. Uso de substâncias psicoativas entre estudantes de enfermagem. Jornal Brasileiro de Psiquiatria. 2007; 56(4):260-66. doi: 10.1590/S0047-20852007000400004.

20. Teixeira RF, Souza RS, Buaiz V, Siqueira MM. Uso de substâncias psicoativas entre estudantes de odontologia da Universidade Federal do Espírito Santo. Revista Ciência \& Saúde Coletiva. 2010; 15 (3): 655-62. doi: $10.1590 /$ S1413-81232010000300007

21. Pereira DS, Souza RS, Buaiz V, Siqueira MM. Uso de substâncias psicoativas entre estudantes de medicina da Universidade Federal do Espírito Santo. Jornal Brasileiro de Pisquiatria. 2008; 57(3):188-95. doi: 10.1590/ S0047-20852008000300006
22. Portugal FB, Souza RS, Buaiz V, Siqueira MM. Uso de drogas por estudantes de Farmácia da Universidade Federal do Espírito Santo. Jornal Brasileiro de Pisquiatria. 2008; 57(2):127-32. doi: 10.1590/ S0047-20852008000200008

23. Nunes JM, Campolina LR, Vieira MA, Caldeira AP. Consumo de bebidas alcoólicas e prática do binge drinking entre acadêmicos da área da saúde. 2012;39(3):94-9. doi: 10.1590/S0101-60832012000300005

24. Santos MVF, Pereira DS, Siqueira MM. Uso de álcool e tabaco entre estudantes de Psicologia da Universidade Federal do Espírito Santo. Jornal Brasileiro de Psiquiatria. 2013; 62(1):22-30. doi: 10.1590/ S0047-20852013000100004

25. Portugal FB, Júnior CC, Siqueira MM. Uso de substâncias psicoativas por futuros educadores. Cad Saúde Coletiva. 2014; 21(3):432-40. doi: 10.1590/ S1414-462X2013000400012

26. Beckert N, Moysés S, Cruz R, Gutoski L, Scarinci I. Características do uso de produtos derivados do tabaco entre universitários do curso de Odontologia em uma Universidade de Curitiba. Rev Odontol UNESP. 2015. doi: 10.1590/1807-2577.10015

27. Picolotto E, Libardoni LFC, Migott AMB, Geib LTC. Prevalência e fatores associados com o consumo de substâncias psicoativas por acadêmicos de enfermagem da Universidade de Passo Fundo. CiêncSaúde Coletiva. 2010;15(3):645-54. doi: 10.1590/ S1413-81232010000300006

28. Rocha NQ, Portella LF, Abreu AMM. Associação entre o consumo de bebidas alcoólicas e tabaco e a religiosidade. Acta Paul Enferm. 2015;28(6). doi: 10.1590/1982-0194201500091.

29. Cardoso FM, Barbosa HA, Costa FM da, Vieira MA, Caldeira AP. Factors associated with practice of binge drinking among students of health. Rev CEFAC. 2015;17(2):475-84. doi: 10.1590/1982-021620158914 30. Funai A, Pillon SC. Uso de bebidas alcoólicas e aspectos religiosos em estudantes de enfermagem. Rev. Eletr. Enf. 2011;13(1):24-9. doi: 10.5216/ree.v13i1.8729 31. Castaño-Perez GA, Calderon-Vallejo GA. Problems associated with alcohol consumption by university students. Rev Latino-Americana de Enfermagem. out. 2014;22(5):739-46. doi: 10.1590/0104-1169.3579.2475 32. Malta DC, Oliveira-Campos M, Prado RR do, Andrade SSC, Mello FCM de, Dias AJR, et al. Psychoactive substance use, family context and mental health among Brazilian adolescents, National Adolescent Schoolbased Health Survey (PeNSE 2012). Revista Brasileira de Epidemiologia. 2014;17(suppl 1):46-61. doi: 10.1590/1809-4503201400050005

33. Uso de bebidas alcoólicas e outras drogas nas rodovias brasileiras e outros estudos/Flavio Pechansky, Paulina do Carmo Arruda Vieira Duarte, Raquel Brandini 
De Boni, organizadores - Porto Alegre: Secretaria Nacional de Políticas sobre Drogas. 2010. 121 p.

34. Mendonça AKRH, Jesus CVF de, Figueiredo MBG de A, Valido DP, Nunes MAP, Lima SO. Consumo de álcool e fatores associados ao binge drinking entre universitárias da área de saúde. Escola Anna Nery. 18 de dezembro de 2017;22(1). doi: 10.1590/2177-9465-EAN-2017-0096

35. Bedendo A, Andrade ALM, Opaleye ES, Noto AR. Binge drinking: a pattern associated with a risk of problems of alcohol use among university students. Revista Latino-Americana de Enfermagem. 2017;25(0). doi: 10.1590/1518-8345.1891.2925

36. Fiori FS, Faria NMX, Meucci RD, Fassa AG. Prevalência e fatores associados ao tabagismo em fumicultores do Sul do Brasil. Caderno de saúde pública. 2016;32(7):1-9. doi: 10.1590/0102-311X00123115

37. Lucchetti G, Peres MF, Lucchetti AL, Koenig HG. Religiosity and tobacco and alcohol use in a Brazilian shantytown. Subst Use Misuse. 2012; 47:837-46. doi: 10.3109/10826084.2012.673142

38. Whooley MA, Boyd AL, Gardin JM, Williams DR. Religious involvement and cigarette smoking in young adults: the CARDIA study (Coronary Artery Risk Development in Young Adults) study. Arch In - tern Med. 2002; 162:1604-10. doi: 10.1001/ archinte.162.14.1604

39. Bezerra J, Barros MVG, Tenório MCM, Tassitano RM, Barros SSH, Hallal PC. Religiosidade, consumo de bebidas alcoólicas e tabagismo em adolescentes. Revista Panamericana Salud Publica. 2009; 26(5):440-6.

40. Dalgalarrondo P, Soldera MA, Corrêa Filho HR, Silva CAM. Religião e uso de drogas por adolescentes. Revista Brasileira de Psiquiatria. 2004 Jun;26(2):82-90. doi: 10.1590/S1516-44462004000200004

41. Guimarães ABP, Okabe I, Scarinci IC. A influência da família na iniciação e não utilização de produtos derivados do tabaco: diferenças de gênero. Espaço para a Saúde-Revista de Saúde Pública do Paraná. 2017;18(2):70-76.

42. Guimarães ABP, Hochgraf PB, Brasiliano S, Ingberman YK. Aspectos familiares de meninas adolescentes dependentes de álcool e drogas. Revista de Psiquiatria Clínica. 2009;36(2):69-74.

43. Malta DC, Porto DL, Melo FCM, Monteiro RA, Sardinha LMV, Lessa BH. Família e proteção ao uso de tabaco, álcool e drogas em adolescentes, Pesquisa
Nacional de Saúde dos Escolares. Revista Brasileira de Epidemiologia. 2011 Sep;14(suppl 1):166-77. doi: 10.1590/S1415-790X2011000500017

44. Nunes De Lima JR, Araújo de Medeiros Pereira AK, Cosme do Nascimento EG, Alchieri JC. Percepção do acadêmico de enfermagem sobre o seu processo de saúde/doença durante a graduação. Saúde \& Transformação Social/Health \& Social Change. 2013;4(4).

45. Cui X, Rockett IR, Yang T, Cao R. Work stress, life stress, and smoking among rural-urban migrant workers in China. BMC Public Health. 2012;12(1). doi: 10.1186/1471-2458-12-979.

\section{Contribuição dos autores}

Concepção e desenho da pesquisa: Marina Coelho de Pinho, Rayane Cristina Faria de Souza, Flavia Batista Portugal, Marluce Mechelli de Siqueira. Obtenção de dados: Marina Coelho de Pinho, Rayane Cristina Faria de Souza. Análise e interpretação dos dados: Marina Coelho de Pinho, Flavia Batista Portugal, Marluce Mechelli de Siqueira. Análise estatística: Marina Coelho de Pinho, Flavia Batista Portugal. Redação do manuscrito: Marina Coelho de Pinho, Rayane Cristina Faria de Souza, Flavia Batista Portugal, Marluce Mechelli de Siqueira. Revisão crítica do manuscrito quanto ao conteúdo intelectual importante: Marina Coelho de Pinho, Rayane Cristina Faria de Souza, Flavia Batista Portugal, Marluce Mechelli de Siqueira.

Todos os autores aprovaram a versão final do texto.

Conflito de interesse: os autores declararam que não há conflito de interesse. 